\title{
Small-Angle Neutron Scattering Reveals Pressure-Dependent Methane Trapping in Shale
}

\author{
Chelsea W. NeiL ${ }^{1}$, Mohamed Mehana ${ }^{1}$, Rex P. \\ HJELM $^{2}$, MARILYN E. HAWLEY ${ }^{2}$, YIMIN MAO ${ }^{3}$, HARI \\ VISWANATHAN $^{1}$, QINJUN KANG ${ }^{1}$, AND HONGWU XU ${ }^{1}$ \\ ${ }^{1}$ Earth and Environmental Sciences Division, Los Alamos \\ National Laboratory, Los Alamos, NM 87545, USA \\ ${ }^{2}$ Materials Science and Technology Division, Los Alamos \\ National Laboratory, Los Alamos, NM 87545, USA \\ ${ }^{3}$ NIST Center for Neutron Research, National Institute of \\ Standards and Technology, Gaithersburg, MD 20899, \\ USA
}

Economical and sustainable hydrocarbon production from unconventional reservoirs necessitates improved understanding of the physical and chemical mechanisms governing fluid transport at nanoscale. For both gas and oil, production declines hyperbolically upon well establishment due to limited extents of matrix diffusion or desorption of hydrocarbons from the shale matrix, resulting in recovery rates of $<10 \%$ for oil and around $20 \%$ for gas. Understanding these transport processes is further complicated by the predominance of nanoconfinement effects within the shale matrix, affecting fluid behavior in ways that are hard to predict and require further experimental validation. In the current study, we integrated molecular dynamics (MD) modeling with smallangle neutron scattering (SANS), a powerful technique capable of characterizing methane behavior within shale nanopores in situ at elevated pressures. Our results illuminated a new mechanism whereby higher peak pressures led to the trapping of dense, liquid-like methane in smaller nanopores. These pores are both abundant in shale rock and believed to host the majority of target hydrocarbons, giving our new findings critical implications for how pressure management can applied to maximize hydrocarbon recovery. 